\title{
Transplantation of Olfactory Mucosa as a Scaffold for Axonal Regeneration Following Spinal Cord Contusion in Rats
}

\author{
Koichi Iwatsuki $^{1}$, Toshiki Yoshimine ${ }^{1}$, Yoshiyuki Sankai ${ }^{2}$, Masao Umegaki ${ }^{1}$, Yu-Ichiro Ohnishi ${ }^{1}$, \\ Masahiro Ishihara ${ }^{1}$, Takashi Moriwaki ${ }^{1}$, Noriko Oda ${ }^{1}$ \\ ${ }^{1}$ Department of Neurosurgery, Medical School, Osaka University, Osaka, Japan; ${ }^{2}$ Faculty of Engineering, Information and Systems, \\ University of Tsukuba, Tsukuba, Japan. \\ Email: kiwatsuki@nsurg.med.osaka-u.ac.jp
}

Received February $28^{\text {th }}, 2013$; revised March $30^{\text {th }}, 2013$; accepted May $1^{\text {st }}, 2013$

Copyright (C) 2013 Koichi Iwatsuki et al. This is an open access article distributed under the Creative Commons Attribution License, which permits unrestricted use, distribution, and reproduction in any medium, provided the original work is properly cited.

\begin{abstract}
Object: The inability of the spinal cord to regenerate after SCI is due to the extremely limited regenerative capacity of most central nervous system (CNS) axons, along with the hostile environment of the adult CNS, which does not support axonal growth. It seems that for successful axonal regeneration to take place, a supportive local environment is required after the injury. We have previously reported that transplantation of the olfactory mucosa is effective in restoring functional recovery in rats following spinal cord transaction. In this study, we examined histological features of olfactory mucosa grafts in rats subjected to a spinal cord contusion protocol. Respiratory mucosa was utilized as a control, as we have previously found that respiratory mucosa does not support neuronal generation. Methods: The rats spinal cords were crash-injured by dropping a 10-g metal rod from a height of $7.5 \mathrm{~cm}$, and a couple of weeks later, the injury sites were exposed, and both olfactory and respiratory mucosae were inserted into the posterior sulcuses of the spinal cord. The each number of olfactory and mucosa transplanted rats were five. The Basso, Beattie, and Bresnahan (BBB) score was observed. Immunohistochemical study for neurofilament was performed. Results: Olfactory mucosa transplanted rats following spinal cord injury can support at least partial hind limb motor recovery compared with respiratory mucosa transplanted rats and we identified numerous axons surrounding the transplanted olfactory mucosa cells, and penetrating the olfactory mucosa at the transplant site. Conclusion: Olfactory mucosa might be a suitable scaffold for axonal regeneration.
\end{abstract}

Keywords: Olfactory Mucosa; Spinal Cord Injury; Transplantation; Scaffold

\section{Introduction}

Traumatic spinal cord injury (SCI) is relatively common, and can result in severe damage leading to partial or complete loss of motor and sensory function caudal to the level of injury. This occurs as a result of severing of descending and ascending fiber tracts. One of the most devastating permanent complications following SCI is paraplegia, management of which has been a constant challenge in clinical medicine. Facilitating restoration of tract structure, and with it recovery of function, after SCI is of great interest to neuroscientists. The inability of the spinal cord to regenerate after SCI is due to the extremely limited regenerative capacity of most central nervous system (CNS) axons, along with the hostile environment of the adult CNS, which does not support ax- onal growth. After an SCI, astroglial scarring occurs within lesioned areas [1]. It has been shown that axonal regeneration is in fact initiated in the injured spinal cord but that it is blocked by glialscar formation [2]. It seems that for successful axonal regeneration to take place, a supportive local environment is required from an early stage after the injury. Recently [3], a team reported partial success in bridging the ends of the spinal cord after a complete resection using grafts of smooth muscle, peripheral nerve [4], fetal brain cells [5], semi-fluid collagen material [6], and embryonic spinal cord segments in the neonatal rat. These experiments suggest that regeneration of spinal nerve fibers across a spinal cord defect could be possible, under favorable conditions. To date, there have been very few studies regarding events that occur in the early stages of autografts transplantation. 
We have previously reported that grafts of the olfactory mucosa are effective in restoring functional recovery in rats following spinal cord transaction, with histological evidence of neuronal regeneration [7-9]. In the present study, we examined histological features of olfactory mucosa grafts in rats subjected to a spinal cord contusion protocol. Respiratory mucosa was utilized as a control, as we have previously found that respiratory mucosa does not support neuronal generation.

\section{Materials and Methods}

Spinal cord injury model Male Sprague-Dawley rats, weighing $250-300 \mathrm{~g}$, were anesthetized using a pentobarbiturate sodium/atropine mixture $(5 / 5 \mathrm{mg} / \mathrm{kg}$, intraperitoneally). Rectal temperature was maintained at $37^{\circ} \mathrm{C}$ $\pm 0.5^{\circ} \mathrm{C}$ using a heating pad. A laminectomy was performed at the thoracic (Th) $8-9$ vertebrae using a microsurgery bone rongeur to expose the spinal cord without touching it. The spinal cord, covered by the dura mater, was crush-injured by dropping a 10-g metal rod from a height of $7.5 \mathrm{~cm}$ using a New York University (NYU) impactor. Although crush injury is commonly simulated by dropping a weight from a height of $2.5-5.0 \mathrm{~cm} 4$ mild or moderate injuries tend to produce high rates of spontaneous locomotor recovery in controls. We dropped a rod from a height of $7.5 \mathrm{~cm}$ to cause severe crush injuries. Dissection and preparation of olfactory and respiratory mucosa Rats were deeply anesthetized using sodium pentobarbital $(100 \mathrm{mg} / \mathrm{kg})$ and sacrificed by decapitation. The nasal septum was freed by removing the lower jaw, upper teeth, and nasal turbinates. Both olfactory and respiratory mucasae were identified on the septum. The olfactory mucosa is located in the dorsocaudal portion and is easily identifiable by the yellowish appearance of its surface. The respiratory mucosa is located ventrorostral to the olfactory mucosa and identified by the grayish color of its surface. Each mucosa was carefully dissected to exclude the border region between the mucosae in order to avoid cross-contamination between the 2 types. Transplantation of olfactory and respiratory mucosa A couple of weeks after injury, the injury site was exposed, and the posterior sulcuses of the spinal cord were opened. Both olfactory and respiratory mucosae were divided into approximately $0.5-1.0-\mathrm{mm}$ sections.

Next, 2 - 3 sections of the olfactory and respiratory mucosae were gently inserted into the sulcuses respectively. The each number of olfactory and mucosa transplanted rats were five. The wound was sealed by suturing the muscle and the skin overlying the exposed spine. Behavioral assessment The Basso, Beattie, and Bresnahan (BBB) score is an operationally defined 21-point scale. It is designed to assess the degree of hind limb locomotor recovery following impact injury to the tho- racic spinal cord in rats [10]. In the present study, the BBB score in each animal was determined by 2 independent observers, who were blinded to the purpose and other protocols of this study. The scores were averaged and compared between the 2 groups using the Student's $t$ test (unpaired). Statistical significance was set at $\mathrm{p}<0.05$. Preparation of tissue for histology and immunohistochemistry for immunohistochemical examination, 3 rats from each of the transplantation groups were sacrificed 8 weeks after the transplantation. Rats were deeply anesthetized by an intraperitoneal injection of sodium pentobarbital $(100 \mathrm{mg} / \mathrm{kg})$, and perfused intracardially with 50 $\mathrm{ml}$ PBS, followed by $200 \mathrm{ml}$ of a fixative containing $2 \%$ paraformaldehyde in $0.1 \mathrm{M}$ phosphate buffer, $\mathrm{pH}$ 7.4. Specimens were processed using a standard procedure for embedding in OCT compound, and cut horizontally into 7- $\mu \mathrm{m}$-thick frozen sections with a cryostat (CM1510S; Leica). Frozen sections were mounted on coated glass slides.

For histological examination, horizontal sections were stained with hematoxylin and eosin (HE), and used for observing blood vessels, and measuring the volume of cavities in the spinal cord. For immunohistochemistry, sections were washed 3 times with PBS, and blocked with a $0.1 \%$ bovine serum albumin solution containing $0.1 \%$ Tween 20 in PBS for 30 min. Sections were then incubated overnight in a solution containing primary antibodies as follows: anti-p75NGFR (Chemicon, Cat. No. MAB365; $1: 500$ in 0.1 M PBS pH 7.4) for olfactory ensheathing cells, anti-glial fibrillary acidic protein (GFAP) monoclonal antibody (1:300; Sigma) for astro- cytes, and anti-neurofilament $200 \mathrm{kD}$ rabbit polyclonal antibody (1:100; Chemicon) for axons. After washing, the sections were incubated overnight with secondary antibodies as follows: FITC- or Cy-3-labeled anti-mouse IgG antibody (1:1000; Amersham Biosciences) for astrocytes, or Cy-3labeled anti-rabbit $\operatorname{IgG}$ antibody (1:1000; Amersham Biosciences) for axons. Sections were then mounted and examined by a fluorescence microscope (Axio Imager MI; Carl Zeiss).

All experimental procedures were approved by the Animal Ethics Committees of the Osaka University Medical School.

\section{Results}

The averaged BBB scores of the olfactory mucosa transplanted rats $(\mathrm{n}=5)$ were $3.13 \pm 1.12,5.25 \pm 1.21,6.88 \pm$ 1.34 , and $10.83 \pm 1.23$, measured $1,2,4$, and 8 weeks after transplantation, respectively. The averaged BBB scores of the respiratory mucosa transplanted rats $(n=5)$, measured over the same time frame, were $2.2 \pm 0.84,2.8$ $\pm 1.15,3.5 \pm 1.02$, and $4.0 \pm 0.71$. These data indicate that the recovery of hind limb movement in the olfactory 
mucosa transplanted rats improved significantly in comparison to the control, respiratory mucosa transplanted rats $(\mathrm{p}<0.05)$ (Figure 1). In the histological assessment, expression of neurofilament was observed strongly at the injury site in the olfactory mucosa transplanted rats (Figures 2 and 3). The numerous fibers that were strongly stained with neurofilament were surrounding the GFP-positive cells and penetrating the transplanted olfactory mucosa (Figure 3). In contrast, there were no apparent neurofilament stained fibers at the marginal spinal cord of the respiratory mucosa transplanted rats (Figure 4).

\section{Discussion}

Injuries to the central nervous system (CNS) in humans are usually associated with a low degree of neurological recovery and, in the majority of cases, life-long debilitation. This lack of recovery, however, is not due to any intrinsic inability of CNS axons to regenerate; rather, the environment of the CNS is strongly inhibitory to axonal regeneration. Following SCI, astroglial scars form within lesioned areas of the spinal cord [1]. Although the majo-
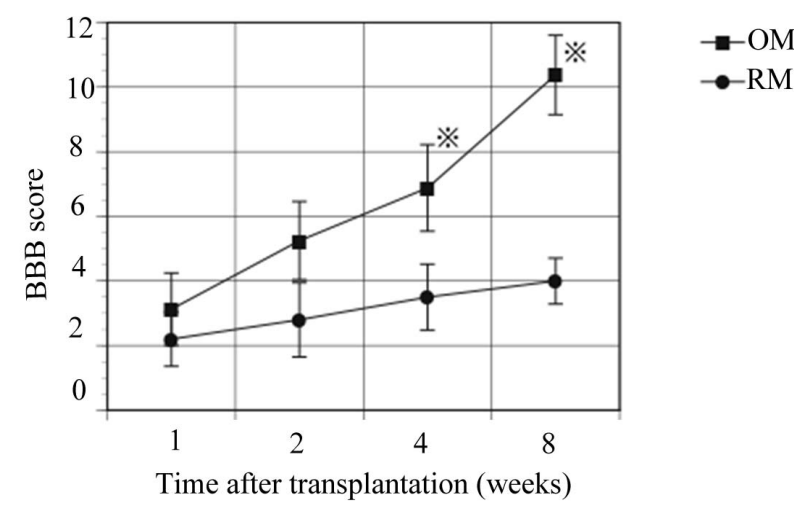

Figure 1. A significantly greater degree of functional recovery as measured by hindlimb usage was observed in the olfactory mucosa transplanted rats (OM) compared with the respiratory mucosa transplanted rats (RM) 4 weeks after the transplantation (※). BBB: Basso, Beattie, and Bresnahan locomotor rating scale.

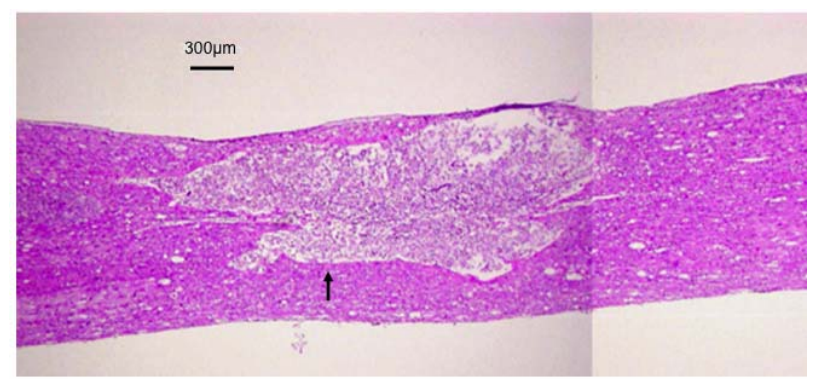

Figure 2. Histology (HE). A transplanted mucosa (indicated by an arrow) is recognizable in the contused spinal cord.

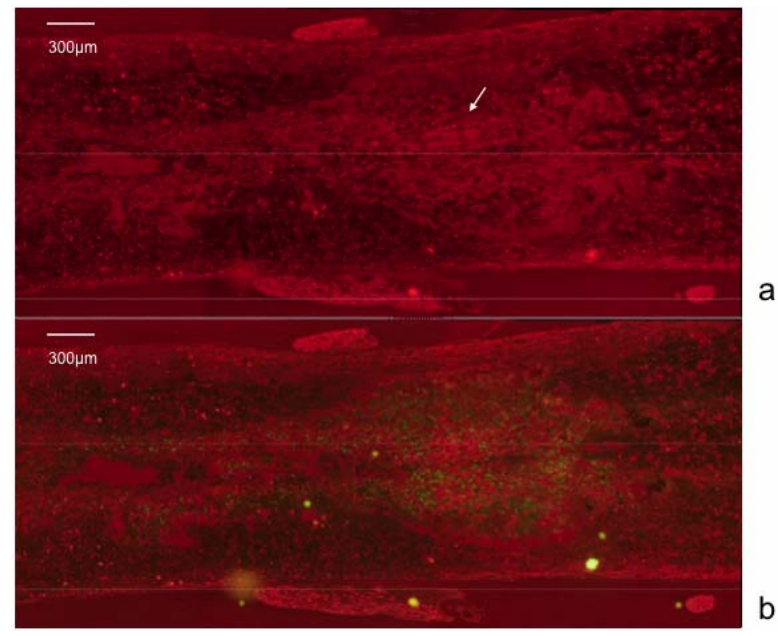

Figure 3. Immunohistological study. Numerous fibers (arrow), strongly stained with neurofilament, are seen penetrating the transplanted olfactory mucosa (a); The fibers surround the GFP-positive cells (b).

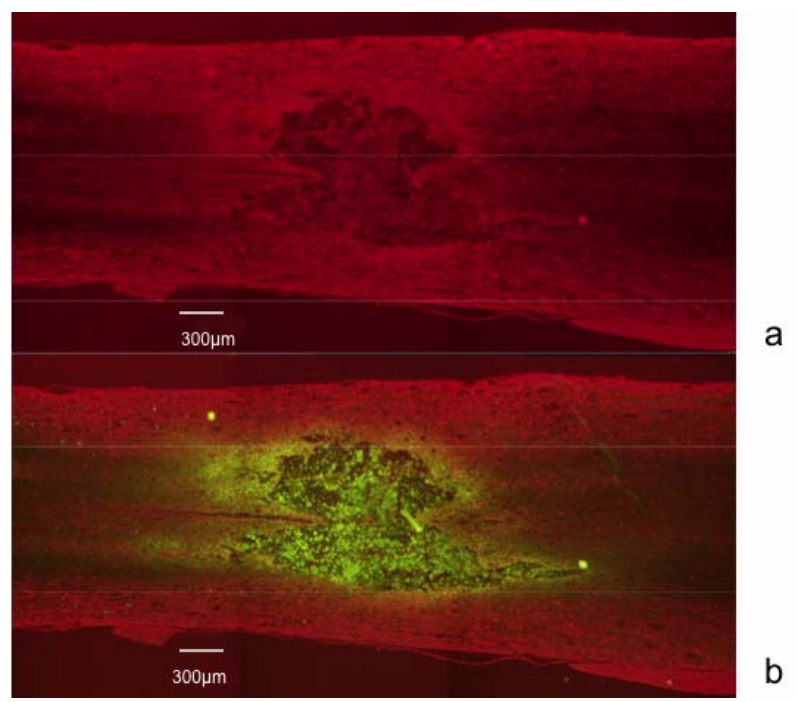

Figure 4. Immunohistological study. No apparent fibers stained with neurofilament are found in the respiratory mucosa (GFP-positive) transplanted spinal cord.

rity of known inhibitors of neurite outgrowth are myelin membrane proteins, equally potent inhibitors have also been identified in astroglial scars, for example, chondroitin sulfate proteoglycans and semaphoring $3 \mathrm{~A}$.

Manipulating the local environment in order to provide a favorable scaffold, supportive of axonal regeneration, is one of the more promising strategies for treatment of SCI. Spinal cord reconstruction using implantation of cells from various sources has been gaining attention in recent years $[11,12]$. Neuronal stem cells have the potential to differentiate into both neuronal and glial cells, and are therefore prime candidates for cell replacement therapy following CNS injury. Neuronal stem cells constitutively 
secrete significant quantities of several neurotrophic factors that act to support host axonal regeneration after SCI [13]. Partial restoration of function after contusion of the spinal cord has been accomplished by injecting neu$\mathrm{ral} / \mathrm{glial}$ precursors (NSCs), differentiated in vitro from mouse embryonic stem cells, into the lesion 9 days after injury [14]. However, implantation of NSCs alone did not produce any significant restorative effect because the majority of the NSCs grafted into the spinal cord differentiated with an astrocytic phenotype $[13,15]$. Although astrocytes can secrete neurotrophic factors and limit the extent of the inflammatory reaction, extensive astroglial scarring within the lesioned area blocks axon growth.

However, one of the major disadvantages associated with implantation or injection of cells alone is the limited proportion of viable cells surviving in the injury site after the procedure, as cells tend to migrate away from the injury site [16]. To achieve significant functional reconstruction of the spinal cord after spinal cord injuries, it is either necessary to populate lesion sites with tissue-specific, regeneration-competent cells that replace or rescue dying cells, or to activate endogenous neural progenitor cells that do likewise [17]. In this study, numerous neurofilaments were observed strongly in the transplanted olfactory mucosa. Unlike respiratory mucosa, it permits axonal regeneration after SCI and therefore may be an appropriate scaffold on which to reconstruct axons. Indeed, the olfactory mucosa is an excellent autologous source of adult neuronal precursor cells. The neurons and the sustentacular cells there renew themselves constantly throughout life by proliferation of basal global stem cells [18-20]. Furthermore, the mucosa contains olfactory ensheathing cells, which have previously been the subject of much attention for their potential in the repair of spinal cord injuries [21-24]. Recent studies of spinal cord axon regeneration have reported good long-term results using various types of tissue scaffolds [25-27]. Olfactory tissue would allow autologous transplantation, is easily accessible, and can be obtained by a simple biopsy that is performed through the external nares [28]. These considerations, combined with the results of the present study, make nasal mucosa an attractive potential scaffold for axonal regeneration.

\section{Conclusions}

As we have previously reported, olfactory mucosa transplantation following spinal cord injury can support at least partial hind limb motor recovery. In this study, we identified numerous axons surrounding the transplanted cells, and penetrating the mucosa at the transplant site without marginal spinal white matter. Olfactory mucosa might therefore be a more suitable scaffold for axonal regeneration than white matter, which contains inhibiting factors for axonal regeneration in the spinal cord.

This study was supported by the "Center for Cybernics Research-World Leading Human-Assistive Technology Supporting a Long-Lived and Healthy Society" granted through the "Funding Program for World-Leading Innovative $\mathrm{R} \& \mathrm{D}$ on Science and Technology (FIRST Program)," initiated by the Council for Science and Technology Policy.

\section{REFERENCES}

[1] J. W. McDonald and C. Sadowsky, "Spinal-Cord Injury," Lancet, Vol. 359, No. 9304, 2002, pp. 417-425. doi:10.1016/S0140-6736(02)07603-1

[2] M. T. Filbin, "Axon Regeneration: Vaccinating against Spinal Cord Injury," Current Biology, Vol. 10, No. 3, 2000, pp. R100-R103. doi:10.1016/S0960-9822(00)00302-X

[3] S. Yoshii, M. Oka, M. Shima, M. Akagi and A. Taniguchi, "Bridging a Spinal Cord Defect Using Collagen Filament," Spine, Vol. 28, 2003, pp. 2346-2351.

[4] L. G. Nygren, L. Olson and A. Seiger, "Mnoaminergic Reinnervation of the Transected Spinal Cord by Homologous Fetal Brain Grafts," Brain Research, Vol. 129, No. 2, 1977, pp. 227-235. doi:10.1016/0006-8993(77)90003-8

[5] G. Galabov, "Regeneration of Sectioned Spinal Cord by Implanatation of a Peripheral Nerve," Doklady Bolgarskoŭ Akademii Nauk, Vol. 19, 1966, pp. 449-452.

[6] J. Cde la Torre, "Catecholamine Fiber Regeneration across a Collagen Bioimplant after Spinal Cord Transection," Brain Research Bulletin, Vol. 9, No. 1-6, 1982, pp. 545-552. doi:10.1016/0361-9230(82)90162-9

[7] M. Aoki, H. Kishima, K. Yoshimura, M. Ishihara, M. Ueno, K. Hata, et al., "Limited Functional Recovery in Rats with Complete Spinal Cord Injuryafter Transplantation of Whole-Layer Olfactory Mucosa: Laboratory Investigation," Journal of Neurosurgery: Spine, Vol. 12, No. 2, 2010, pp. 122-130. doi:10.3171/2009.9.SPINE09233

[8] K. Iwatsuki, T. Yoshimine, H. Kishima, M. Aoki, K. Yoshimura, M. Ishihara, et al., "Transplantation of Olfactory Mucosa Following Spinal Cord Injury Promotes Recovery in Rats," Neuroreport, Vol. 19, 2008, pp. 12491252. doi:10.1097/WNR.0b013e328305b70b

[9] J. Nakayama, T. Takao, H. Kiuchi, K. Yamamoto, S. Fukuhara, Y. Miyagawa, et al., "Olfactory Mucosal Transplantation after Spinal Cord Injury Improves Voiding Efficiency by Suppressing Detrusor-Sphincter Dyssynergia in Rats," The Journal of Urology, Vol. 184, No. 2, 2010, pp. 775-782. doi:10.1016/j.juro.2010.03.105

[10] D. M. Basso, M. S. Beattie and J. C. Bresnahan, "A Sensitive and Reliable Locomotor Rating Scale for Open Field Testing in Rats," Journal of Neurotrauma, Vol. 12, No. 1, 1995, pp. 1-21. doi:10.1089/neu.1995.12.1

[11] N. Keyvan-Fouladi, G. Raisman and Y. Li, "Functional Repair of the Corticospinal Tract by Delayed Transplan- 
tation of Olfactory Ensheathing Cells in Adult Rats," The Journal of Neuroscience, Vol. 23, 2003, pp. 9428-9434.

[12] Y. Ogawa, K. Sawamoto, T. Miyata, S. Miyao, M. Watanabe, M. Nakamura, et al., "Transplantation of in $\mathrm{Vi}$ tro-Expanded Fetal Neural Progenitor Cells Results in Neurogenesis and Functional Recovery after Spinal Cord Contusion Injury in Adult Rats," Journal of Neuroscience Research, Vol. 69, No. 6, 2002, pp. 925-933. doi:10.1002/jnr.10341

[13] S. S. Han, D. Y. Kang, T. Mujtaba, M. S. Rao and I. Fischer, "Grafted Lineage-Restricted Precursors Differentiate Exclusively into Neurons in the Adult Spinal Cord," Experimental Neurology, Vol. 177, No. 2, 2002, pp. 360375. doi:10.1006/exnr.2002.7995

[14] J. W. McDonald, X. Z. Liu, Y. Qu, S. Liu, S. K. Mickey, D. Turetsky, et al., "Transplanted Embryonic Stem Cells Survive, Differentiate and Promote Recovery in Injured Rat Spinal Cord," Nature Medicine, Vol. 5, 1999, pp. 1410-1412. doi:10.1038/70986

[15] Q. L. Cao, Y. P. Zhang, R. M. Howard, W. M. Walters, P. Tsoulfas, S. R. Whittemore, et al., "Pluripotent Stem Cells Engrafted into the Normal or Lesioned Adult Rat Spinal Cord Are Restricted to a Glial Lineage," Experimental Neurology, Vol. 167, No. 1, 2001, pp. 48-58. doi:10.1006/exnr.2000.7536

[16] S. Okada, K. Ishii, J. Yamane, A. Iwanami, T. Ikegami, H. Katoh, et al., "In Vivo Imaging of Engrafted Neural Stem Cells: Its Application in Evaluating the Optimal Timing of Transplantation for Spinal Cord Injury," FASEB Journal, Vol. 19, 2005, pp. 1839-1841.

[17] D. L. Stocum, "Stem Cells in CNS and Cardiac Regeneration," Advances in Biochemical Engineering, Vol. 93, 2005, pp. 135-159. doi:10.1007/b99969

[18] A. L. Calof, A. Bonnin, C. Crocker, S. Kawauchi, R. C. Murray, J. Shou, et al., "Progenitor Cells of the Olfactory Receptor Neuron Lineage," Microscopy Research and Technique, Vol. 58, No. 3, 2002, pp. 176-188. doi:10.1002/jemt.10147

[19] A. M. Cunningham, P. B. Manis, R. R. Reed and G. V. Ronnett, "Olfactory Receptor Neurons Exist as Distinct Subclasses of Immature and Mature Cells in Primary Culture," Neuroscience, Vol. 93, No. 4, 1999, pp. 13011312. doi:10.1016/S0306-4522(99)00193-1
[20] F. J. Roisen, K. M. Klueber, C. L. Lu, L. M. Hatcher, A. Dozier, C. B. Shields, et al., "Adult Human Olfactory Stem Cells," Brain Research, Vol. 890, No. 1, 2001, pp. 11-22. doi:10.1016/S0006-8993(00)03016-X

[21] S. C. Barnett and J. S. Riddell, "Olfactory Ensheathing Cells (OECs) and Thetreatment of CNS Injury: Advantages and Possible Caveats," Journal of Anatomy, Vol. 204, No. 1, 2004, pp. 57-67. doi:10.1111/j.1469-7580.2004.00257.x

[22] Y. Li, P. M. Field and G. Raisman, "Repair of Adult Rat Corticospinal Tract by Transplants of Olfactory Ensheathing Cells," Science, Vol. 277, No. 5334, 1997, pp. 2000-2002. doi:10.1126/science.277.5334.2000

[23] A. Ramon-Cueto and F. Valverde, "Olfactory Bulb Ensheathing Glia: A Unique Cell Type with Axonal GrowthPromoting Properties," Glia, Vol. 14, No. 3, 1995, pp. 163-173. doi:10.1002/glia.440140302

[24] P. M. Smith, A. Lakatos, S. C. Barnett, N. D. Jeffery, R. J. Franklin, et al., "Cryopreserved Cells Isolated from the Adult Canine Olfactory Bulb Are Capable of Extensive Remyelination Following Transplantation into the Adult Rat CNS," Experimental Neurology, Vol. 176, No. 2, 2002, pp. 402-406. doi:10.1006/exnr.2002.7936

[25] K. Kataoka, Y. Suzuki, M. Kitada, T. Hashimoto, H. Chou, H. Bai, et al., "Alginate Enhances Elongation of Early Regenerating Axons in Spinal Cord of Young Rats," Tissue Engineering, Vol. 10, No. 3-4, 2004, pp. 493-504. doi:10.1089/107632704323061852

[26] M. J. Moore, J. A. Friedman, E. B. Lewellyn, S. M. Mantila, A. J. Krych, S. Ameenuddin, et al., "MultipleChannel Scaffolds to Promote Spinal Cord Axon Regeneration," Biomaterials, Vol. 27, No. 3, 2006, pp. 419-429. doi:10.1016/j.biomaterials.2005.07.045

[27] S. Stokols and M. H. Tuszynski, "Freeze-Dried Agarose Scaffolds with Uniaxial Channels Stimulate and Guide Linear Axonal Growth Following Spinal Cord Injury," Biomaterials, Vol. 27, No. 3, 2006, pp. 443-451. doi:10.1016/j.biomaterials.2005.06.039

[28] F. Feron, C. Perry, J. J. McGrath and A. Mackay-Sim, "New Techniques for Biopsy and Culture of Human Olfactory Epithelial Neurons," Archives of Otolaryngology-Head and Neck Surgery, Vol. 124, 1998, pp. 861866. 